\title{
Coronary Endothelial Dysfunction and Non-Contrast Multidetector Computed Tomography
}

\author{
Akira Kurata, MD; Hiroshi Higashino, MD; Teruhito Mochizuki, MD
}

$\mathbf{E}$ ndothelial dysfunction, which occurs in the earliest stage of arteriosclerosis, is considered to be a predictor of the consequences of atherosclerosis-associated determinants, ${ }^{1-3}$ coronary artery disease $(\mathrm{CAD}),{ }^{3}$ and future cardiovascular events. ${ }^{3-5}$

\section{Article p 160}

Previous authors have evaluated endothelial dysfunction after intracoronary infusion of acetylcholine by Doppler flow wire (coronary blood flow) or invasive coronary angiography (coronary artery vasodilation). ${ }^{4-6}$ More recent reports have shown that non-invasive assessment of myocardial blood flow (MBF) by positron emission tomography (PET) can effectively evaluate coronary endothelial dysfunction using a coldpressor test $(\mathrm{CPT})^{2,7}$ or pharmacological stimuli. ${ }^{3} \mathrm{CPT}$ induces coronary vasodilation directly through a mechanism involving the sympathetic nervous system ( $\beta$-adrenergic activation and increased rate-pressure product [RPP]), or indirectly through endothelial-derived nitric oxide signaling., ${ }^{2,7}$ On the other hand, adenosine, which is usually used as a pharmacological stimulus, induces direct vasodilation in resistance vessels and increases MBF independently of myocardial oxygen consumption. ${ }^{8}$

Contrast-enhanced multidetector row computed tomography (MDCT) has been widely used in clinical practice as coronary $\mathrm{CT}$ angiography for the diagnosis of CAD. ${ }^{9}$ In addition, myocardial perfusion CT has been useful in assessing myocardial ischemia in patients with CAD after pharmacological stress-induced MBF changes. ${ }^{10,11}$ Use of a contrast medium and relatively high radiation exposure are still the main limitations of the technique.

In this issue of the Journal, Dunet et $\mathrm{al}^{12}$ propose a unique application of non-contract MDCT to assess changes in the coronary cross-sectional area (CSA) before and during CPT. They report the feasibility of non-contrast, low-dose MDCT in the assessment of coronary vasoreactivity in comparison with ${ }^{82} \mathrm{Rb}$ myocardial perfusion PET. They show that relative changes in the coronary CSA can be assessed by MDCT, reflecting vasodilation during CPT, which correlate to changes in MBF by ${ }^{82} \mathrm{Rb}$ PET. The conclusion rests on the following evidence: (1) MBF significantly increased from $0.88 \pm 0.26 \mathrm{ml} \cdot \mathrm{min}^{-1} \cdot \mathrm{g}^{-1}$ at rest to $1.01 \pm 0.31 \mathrm{ml} \cdot \mathrm{min}^{-1} \cdot \mathrm{g}^{-1}$ during CPT in a PET study (absolute and relative mean increase
MBF: $0.13 \pm 0.18 \mathrm{ml} \cdot \mathrm{min}^{-1} \cdot \mathrm{g}^{-1}$ and $16 \pm 18 \%$, respectively); (2) CPT-induced MBF changes significantly correlated with RPP changes; (3) mean coronary CSA, as measured by MDCT, significantly increased from $20.3 \pm 6.5 \mathrm{~mm}^{2}$ at rest to $22 \pm 6.7 \mathrm{~mm}^{2}$ during CPT (absolute and relative mean change of coronary CSA: $1.7 \pm 1.5 \mathrm{~mm}^{2}$ and $8.9 \pm 7.1 \%$, respectively); and (4) CPTinduced MBF increase moderately correlated with relative changes in coronary CSA.

Although there was statistical significance, a few methodological issues remain to be solved. First, as mentioned above, $\mathrm{CPT}$ and adenosine induce coronary vasodilation through distinct mechanisms. Because adenosine slightly decreases systemic blood pressure, at the same time increases heart rate, ${ }^{13}$ it is not entirely acceptable to compare CPT- and adenosineinduced RPP changes, using the latter as the standard reference. Second, coronary CSA was manually measured. Although the method had excellent reproducibility, parameters should be evaluated with greater objectivity, for instance, by using iterative reconstruction to improve the quality of the image, automated software to detect the contour, and the full-width at half-maximum method. Third, the temporal resolution of MDCT achieved in the present study $(0.35$-s rotation and half reconstruction algorithm) is not robust enough for quantitative assessment of coronary artery dilation along with the CPTinduced higher heart rate. As the authors mention, it would be better to use dual-source $\mathrm{CT}$ with higher temporal resolution. Finally, because of its potent vasodilation effect, adenosine may lead to better results than with CPT.

In addition to coronary calcium scoring ${ }^{14}$ and lipid-rich plaque assessment by computer-aided technique, ${ }^{15}$ non-contrast and low-dose MDCT imaging may be also used to assess the changes of reactive coronary dilation as a surrogate investigation item for coronary endothelial dysfunction in near future. But clinical application of the present study's results may be still limited because of the significant but small amount of change of reactive coronary dilation in comparison with its spatial resolution. This study, however, proposes a unique and potential application of non-contrast and low-dose MDCT coronary imaging.

\section{References}

1. Prior JO, Schindler TH, Facta AD, Hernandez-Pampaloni M, Campisi $\mathrm{R}$, Dahlbom M, et al. Determinants of myocardial blood flow response to cold pressor testing and pharmacologic vasodilation in healthy

The opinions expressed in this article are not necessarily those of the editors or of the Japanese Circulation Society.

Received November 20, 2011; accepted November 20, 2011; released online December 10, 2011

Department of Radiology, Ehime University Graduate School of Medicine, Toon (A.K., T.M.); Department of Radiology, Yotsuba Circulation Clinic, Matsuyama (H.H.), Japan

Mailing address: Akira Kurata, MD, Department of Radiology, Ehime University Graduate School of Medicine, Shitsukawa, Toon 7910295, Japan. E-mail: akuratasan2000@gmail.com

ISSN-1346-9843 doi:10.1253/circj.CJ-11-1342

All rights are reserved to the Japanese Circulation Society. For permissions, please e-mail: cj@j-circ.or.jp 
humans. Eur J Nucl Med Mol Imaging 2007; 34: 20-27.

2. Yoshinaga K, Manabe O, Katoh C, Chen L, Klein R, Naya M, et al. Quantitative analysis of coronary endothelial function with generator-produced 82Rb PET: Comparison with ${ }^{15} \mathrm{O}$-labelled water PET. Eur J Nucl Med Mol Imaging 2010; 37: 2233-2241.

3. Kaufmann PA, Camici PG. Myocardial blood flow measurement by PET: Technical aspects and clinical applications. J Nucl Med 2005; 46: $75-88$

4. Suwaidi JA, Hamasaki S, Higano ST, Nishimura RA, Holmes DR Jr, Lerman A. Long-term follow-up of patients with mild coronary artery disease and endothelial dysfunction. Circulation 2000; 101: $948-$ 954.

5. Zeiher AM, Drexler H, Wollschlaeger H, Saurbier B, Just H. Coronary vasomotion in response to sympathetic stimulation in humans: Importance of the functional integrity of the endothelium. $\mathrm{J} \mathrm{Am} \mathrm{Coll}$ Cardiol 1989; 14: 1181-1190.

6. Schindler TH, Hornig B, Buser PT, Olschewski M, Magosaki N, Pfisterer M, et al. Prognostic value of abnormal vasoreactivity of epicardial coronary arteries to sympathetic stimulation in patients with normal coronary angiograms. Arterioscler Thromb Vasc Biol 2003; 23: $495-501$.

7. Schindler TH, Zhang XL, Prior JO, Cadenas J, Dahlbom M, Sayre J, et al. Assessment of intra- and interobserver reproducibility of rest and cold pressor test-stimulated myocardial blood flow with ${ }^{13} \mathrm{~N}$ ammonia and PET. Eur J Nucl Med Mol Imaging 2007; 34: 11781188.

8. Yoshinaga K, Manabe O, Tamaki N. Physiological assessment of myocardial perfusion using nuclear cardiology would enhance coronary artery disease patient care: Which imaging modality is best for evaluation of myocardial ischemia? (SPECT-side) Circ J 2011; 75: 713-722; discussion 731.

9. Matsumoto N, Sato Y, Yoda S, Nakano Y, Kunimasa T, Matsuo S, et al. Prognostic value of non-obstructive CT low-dense coronary artery plaques detected by multislice computed tomography. $\operatorname{Circ} J$ 2007; 71: $1898-1903$.

10. Kurata A, Mochizuki T, Koyama Y, Haraikawa T, Suzuki J, Shigematsu Y, et al. Myocardial perfusion imaging using adenosine triphosphate stress multi-slice spiral computed tomography: Alternative to stress myocardial perfusion scintigraphy. Circ J 2005; 69: $550-557$.

11. Kido T, Kurata A, Higashino H, Inoue $\mathrm{Y}$, Kanza RE, Okayama $\mathrm{H}$, et al. Quantification of regional myocardial blood flow using first-pass multidetector-row computed tomography and adenosine triphosphate in coronary artery disease. Circ J 2008; 72: 1086-1091.

12. Dunet V, Qanadli SD, Allenbach G, Dabiri A, Mazzolai L, Waeber $\mathrm{B}$, et al. Assessment of coronary vasoreactivity by multidetector computed tomography: Feasibility study with rubidium- 82 cardiac positron emission tomography. Circ J 2012; 76: 160-167.

13. Hatanaka K, Doi M, Hirohata S, Kamikawa S, Kaji Y, Katoh T, at al. Safety of and tolerance to adenosine infusion for myocardial perfusion single-photon emission computed tomography in a Japanese population. Circ J 2007; 71: 904-910.

14. Raggi P, Gongora MC, Gopal A, Callister TQ, Budoff M, Shaw LJ. Coronary artery calcium to predict all-cause mortality in elderly men and women. J Am Coll Cardiol 2008; 52: 17-23.

15. Dey D, Callister T, Slomka P, Aboul-Enein F, Nishina H, Kang X, et al. Computer-aided detection and evaluation of lipid-rich plaque on noncontrast cardiac CT. Am J Roentgenol 2006; 186: S407-S413. 